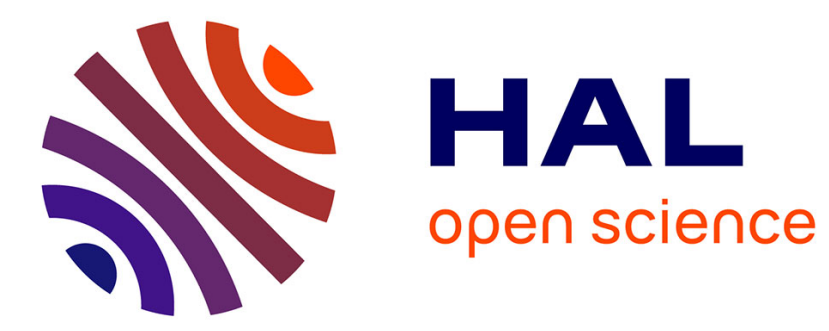

\title{
Answering PICO Clinical Questions: a Semantic Graph-Based Approach
}

\author{
Eya Znaidi, Lynda Tamine, Chiraz Latiri
}

\section{To cite this version:}

Eya Znaidi, Lynda Tamine, Chiraz Latiri. Answering PICO Clinical Questions: a Semantic GraphBased Approach. 15th Conference on Artificial Intelligence in Medicine (AIME 15), Jun 2015, Pavia, Italy. pp. 232-237. hal-01363309

\section{HAL Id: hal-01363309 https://hal.science/hal-01363309}

Submitted on 9 Sep 2016

HAL is a multi-disciplinary open access archive for the deposit and dissemination of scientific research documents, whether they are published or not. The documents may come from teaching and research institutions in France or abroad, or from public or private research centers.
L'archive ouverte pluridisciplinaire HAL, est destinée au dépôt et à la diffusion de documents scientifiques de niveau recherche, publiés ou non, émanant des établissements d'enseignement et de recherche français ou étrangers, des laboratoires publics ou privés. 


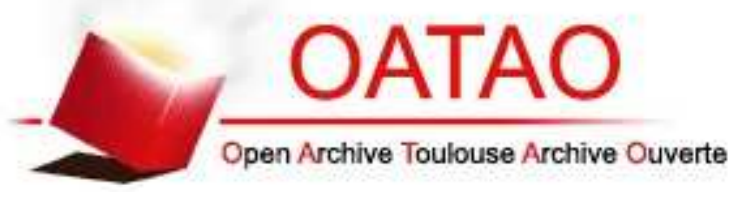

\section{Open Archive TOULOUSE Archive Ouverte (OATAO)}

OATAO is an open access repository that collects the work of Toulouse researchers and makes it freely available over the web where possible.

This is an author-deposited version published in : http://oatao.univ-toulouse.fr/ Eprints ID : 15380

The contribution was presented at AIME 15 :

http://aime15.aimedicine.info/

To cite this version : Znaidi, Eya and Tamine, Lynda and Latiri, Chiraz Answering PICO Clinical Questions: a Semantic Graph-Based Approach. (2015) In: 15th

Conference on Artificial Intelligence in Medicine (AIME 15), 17 June 2015 - 20 June 2015 (Pavia, Italy).

Any correspondence concerning this service should be sent to the repository administrator: staff-oatao@listes-diff.inp-toulouse.fr 


\title{
Answering PICO Clinical Questions: a Semantic Graph-Based Approach
}

\author{
Eya Znaidi ${ }^{1}$, Lynda Tamine ${ }^{1}$, and Chiraz Latiri ${ }^{2}$ \\ 1 IRIT, University Paul Sabatier of Toulouse \\ ${ }^{2}$ Computer Sciences Department, Faculty of Sciences of Tunis
}

\begin{abstract}
In this paper, we tackle the issue related to the retrieval of the best evidence that fits with a PICO (Population, Intervention, Comparison and Outcome) question. We propose a new document ranking algorithm that relies on semantic based query expansion bounded by the local search context to better discard irrelevant documents. Experiments using a standard dataset including 423 PICO questions and more than 1,2 million of documents, show that our aproach is promising.
\end{abstract}

Keywords: Evidence-based-Medicine, PICO Clinical Queries, Medical information retrieval, Semantic Query Expansion

\section{Introduction}

Evidence-Based Medicine (EBM) has been defined as the conscientious, explicit and appropriate use of systematic research findings, in consultation with the patient, with the aim of optimizing the healthcare decision-making process of medical professionals [8]. One major issue faced by the professionals during the daily practice of EBM is the complexity of expressing precise, context-specific clinical queries that better facilitate the identification of the relevant evidence. These works rely heavily on a prior automatic annotation of PICO facets in both queries and documents. Unlikely, our approach (1) relaxes the condition of PICO facet identification in the documents, and (2) abstracts the word-based question formulation by highlighting the overall semantic picture of each question facet. Moreover, each question facet is separately expanded using concepts extracted from top ranked documents issued from the initial retrieval.

The remainder of the paper is structured as follows. In section 2, we first give an overview of related work. Section 3 details our approah for PICO question elicitation and answering. In section 4, we describe the experimental setup and then present and discuss the results obtained using a standard clinical information retrieval dataset. Section 5 concludes the paper.

\section{Related Work}

While some previous work $[1,4,11]$ tackled the issue of PICO element detection, as a prior stage before retrieving relevant documents, other studies, close to our work $[3,2,5]$ focused on the design of retrieval techniques and models that exploit the PICO facets in order to compute the relevance score of documents. To 
achieve this goal, Boudin et al. [3, 2] automatically detected PICO elements in the documents and then revised the basic version of the IR language model [9]. More precisely, the authors revised the word-document weighting shema by taking into account both the distribution of PICO elements in the different passages of the documents and the distributions of the words in the different PICO parts. The experimental evaluation held on a collection of 1.5 million of documents and 423 queries showed that the proposed model yields an improvement of $28 \%$ in mean average precision over state-of-the-art baselines. Demner-Fushman and Lin [5] also proposed an unified framework for both detecting and using the detected PICO elements in the relevance document scoring function $S_{E B M}$. The latter is based on the linear combination of partial relevance scores of the documents considering the three facets of EBM, namely, PICO $\left(S_{P I C O}\right)$, strenght of evidence $\left(S_{S o E}\right)$ and task type $\left(S_{\text {task }}\right)$. For instance, the PICO score relies on a linear combination of $\mathrm{P}, \mathrm{I}, \mathrm{C}$ and $\mathrm{O}$ facet scores considering the word overlap between the document and the question. Experiments carried out on 24 real-world clinical questions show that the approach outperforms a traditional PubMed search.

\section{A Semantic Graph-based Approach for Answering PICO Questions}

We describe in Figure 2 the general algorithm (main and function) for expanding the PICO query and ranking the best evidence to be returned as an answer to the clinician.

- (Main) Steps 1-12: Given a word-based PICO query $Q$, the related annotation $Q_{P I C O}$, the subqueries $Q_{P}, Q_{I C}$ and $Q_{O}$ and the list of $N_{d}$ top ranked documents $D_{N}^{*}$ included in a document collection $C$, the algorithm builds first the semantic sub-graphs $G_{P}, G_{I C}$ and $G_{O}$ after (1) extracting, using our concept method extraction [6] build upon Metamap ${ }^{3}$, the active concepts, respectively Concepts $\left(Q_{P}\right)$, Concepts $\left(Q_{I C}\right)$ and $\operatorname{Concepts}\left(Q_{O}\right)$; each active concept has an importance score $\operatorname{Score}(c)$ that highlights the likelihood of similarity between the concept preferred entry and the query words, (2) building the associated graphs $G_{P}, G_{I C}$ and $G_{O}$ by appending to the active concepts the corresponding hypernyms through terminology function Hyp $G$ processed on medical terminology $T$ until reaching the first common concept. Each returned active concept $c$ is considered at relative level 0 .

- (Main) Steps 13-15: for each sub-graph $G_{P}, G_{I C}$ and $G_{O}$, we build the set of $N_{c}$ concepts to be used for query expansion by applying function Expand $\left(G_{x}\right)$ considering Maxlevel which denotes the maximum level used for query expansion beginning from level 0 .

- (Expand) Steps 1-17: To build the set of candidate concepts Cexpand, we consider each document $d$ in $D_{N}^{*}$ and then (1) extract the set of common

\footnotetext{
${ }^{3}$ http://metamap.nlm.nih.gov
} 


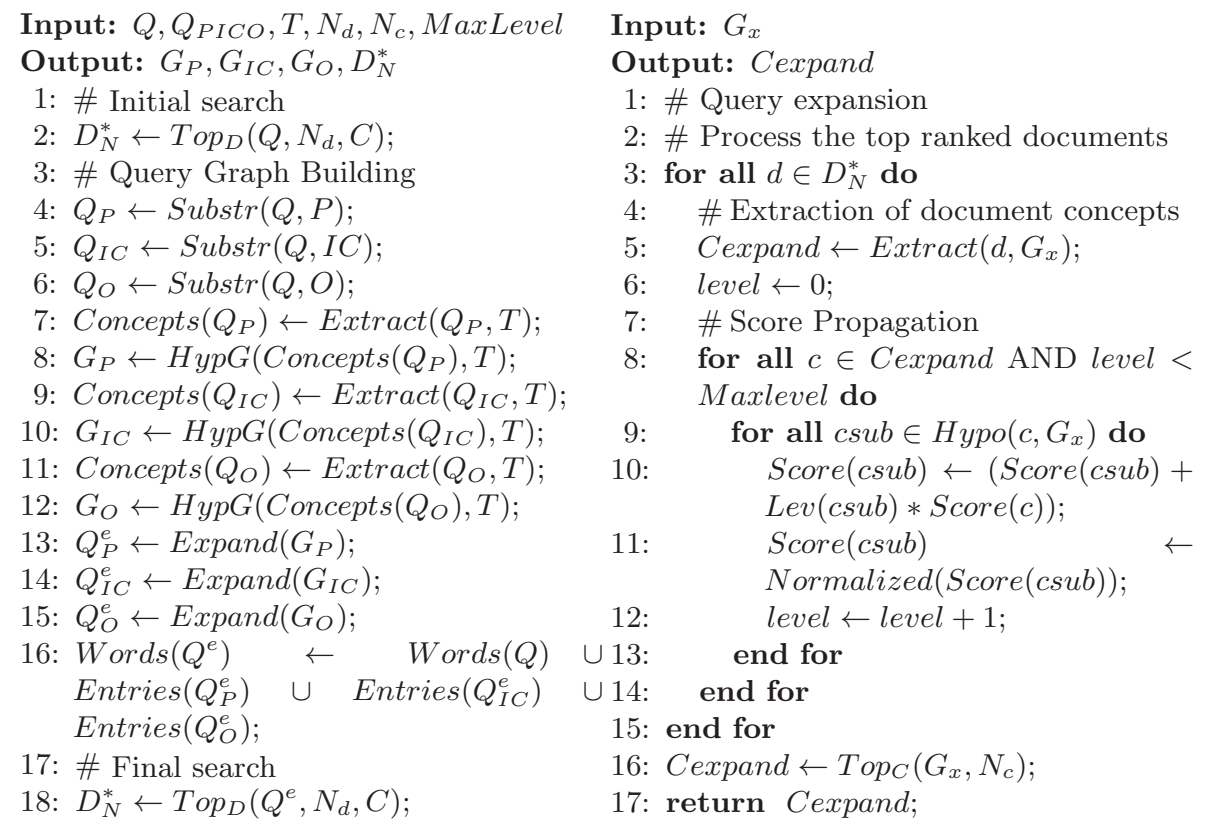

Fig. 1: The document retrieval process

weighted concepts with $G_{x}$ (where $x \in\{P, I C, O\}$ ) using the same concept extraction method [6]; (2) apply a score propagation algorithm that propagates the scores of the active concepts of each query sub-graph $G_{x}$ from level 0 to level Maxlevel by iteratively summing the scores of the hyponym concepts through sub-graph $G_{x}, \operatorname{Hypo}\left(c, G_{x}\right)$. The basic underlying idea is to leverage the importance and the specificity of the concepts by assigning the normalized scores Normalized (Score $(c))$ obtained step by step from less specific concepts to most specific ones, considering their level Lev(c). The final score of a concept reflects its importance in the whole top ranked documents in terms of high specificity and matching degree with documents $D_{N}^{*}$. This fits with our intuition that favors the selection of most specific concepts that better match the search context gathered from the top ranked documents.

- (Main) Steps 16-18: The returned set of $N_{c}$ top weighted concepts Cexpand extracted from each sub-graph $G_{x}$, are used to expand respectively the subqueries $Q_{P}, Q_{I C}$ and $Q_{O}$ (resulting in $Q_{P}^{e}, Q_{I C}^{e}$ and $Q_{O}^{e}$ respectively) by adding to the intial word-based query $Q$ the words belonging to their preferred entries (Entries $\left(Q_{P}^{e}\right)$, Entries $\left(Q_{I C}^{e}\right)$ and Entries $\left(Q_{O}^{e}\right)$ respectively) within terminology $T$. The final expanded query $Q^{e}$ is processed and allows selecting the final list of documents $D_{N}^{*}$ to be returned as an answer to the initial PICO query $Q$. 


\section{Experimental Evaluation}

\subsection{Experimental Setup}

We used the CLIREC dataset which has been built with the specific aim of evaluating clinical information retrieval [3]. Some statistical characteristics of the collection are depicted in Table 1. We used the MeSH terminology which has been widely accepted as the main controlled vocabulary used to index biomedical citations [10]. Each node of the terminology represents a concept node referred to using a preferred entry.

\begin{tabular}{|l|l|}
\hline Number of documents & 1.212 .040 abstracts from PubMed \\
\hline Average document length & 246 words \\
\hline Number of queries & 423 \\
\hline Average number of query keywords & 4.3 words \\
\hline Average PICO query length & 18.7 words \\
\hline Average Number of relevant documents per query & 19 \\
\hline
\end{tabular}

Table 1: CLIREC test collection statistics

For the purpose of evaluating and comparing retrieval effectiveness, we used under version 4.0 of the Terrier search engine $\left.{ }^{4}: 1\right)$ The Mean Average Precision (MAP) measure which is the mean of the AP measure over a set of queries; it is used to provide a single, overall measure of search performance. The performance measures have been computed using the standard TREC-eval tool ${ }^{5}$; 2) We used two state-of-the-art information retrieval models, namely the Okapi probabilistic model (BM25) [7] and the language model (LM) [9]. The Okapi model was parameterized as recommended in the literature: $k 1=1.2, k 3=7$ and $b=0.75$. For the LM, the Dirichlet smoothing method with $\mu=1000$ was used.

\subsection{Results}

We compared the retrieval effectiveness based on $M A P$ of our semantic graphbased document ranking algorithm $G Q E$ with respect to the state-of-the-art ranking models BM25 and $L M$. Table 2 presents the obtained results in terms of the MAP measure and relevant retrieved documents as well as the corresponding pourcents of improvement and significance $t$ values of the statistical t-test. We can see that our model $(G Q E)$ significantly overpasses word-based document ranking approaches $(B M 25, L M)$ from $25,44 \%$ to $27,94 \%$. From these results, we can highlight that our semantic approach allows achieving better results than state-of-the-art word-based IR models that do not specifically take into account the PICO framework; this yiels a credit to our intuition behind question elicitation on the basis of the semantic hidden behind each question facet.

\footnotetext{
${ }^{4}$ http://www.terrier.org

${ }^{5} \mathrm{http} / /$ trec.nist.gov/trec_eval
} 


\begin{tabular}{|l|l|l|l|l|l|}
\hline Model & MAP & \%Change & t & Rel. Ret & \% Change \\
\hline$B M 25$ & 0.1073 & $+25.44 \%$ & $* *$ & 4783 & $+15.28 \%$ \\
$L M$ & 0.1052 & $+27.94 \%$ & $* *$ & 4685 & $+17.69 \%$ \\
\hline$G Q E$ & 0.1346 & - & - & 5514 & - \\
\hline
\end{tabular}

Table 2: Comparison of the semantic graph-based query expansion impact on the retrieval effectiveness. \%Chg: Student test significance over the MAP measure *: $0.01<$ $t \leq 0.05 ;{ }^{* *}: 0.001<t \leq 0.01 ;{ }^{* * *}: t \leq 0.001$.

\section{Conclusion}

In this paper, we presented a novel approach to answer PICO clinical queries. The key underlying idea is to enhance each query facet with the most representative terminological concepts on the basis of a local search context. Moreover, we apply a score propagation algorithm that allows selecting the concepts with higher matching degree over the whole search context and across the different query facets. Experiments on a standard data set highlight that the proposed approach significantly overpasses state-of-the-art IR models. In future, we plan to integrate a weighting facet schema in the document ranking model in order to consider the differences in the importance of the question facets with respect to document relevance.

\section{References}

1. F. Boudin, J.-Y. Nie, J. Bartlett, R. Grad, P. Pluye, and M. Dawes. Combining classifiers for robust pico element detection. BMC Medical Informatics and Decision Making, 10(1):29, 2010.

2. F. Boudin, J. Y. Nie, and M. Dawes. Clinical information retrieval using document and PICO structure. In NAACL HLT, pages 822-830, 2010.

3. F. Boudin, J.-Y. Nie, and M. Dawes. Positional language models for clinical information retrieval. In EMNLP, pages 108-115, 2010.

4. G. Y. Chung. Sentence retrieval for abstracts of randomized controlled trials. $B M C$ Med Inform Decis Mak, 9:10, 2009.

5. D. Demner-Fushman and J. Lin. Answering clinical questions with knowledgebased and statistical techniques. Comput. Linguist., 33(1):63-103, 2007.

6. D. Dinh and L. Tamine. Towards a context sensitive approach to searching information based on domain specific knowledge sources. Web Semantics: Science, Services and Agents on the World Wide Web, 12(0), 2012.

7. S. Robertson and K. S. Jones. Relevance weighting of search terms. Journal of the American Society on Informtion Science and Technology, 27(3), 1976.

8. D. L. Sackett, W. M. C. Rosenberg, J. A. M. Gray, R. B. Haynes, and W. S. Richardson. Evidence based medicine: what it is and what it isn't. BMJ, 312(7023):71-72, 1996.

9. F. Song and W. B. Croft. A general language model for information retrieval. In ACM SIGIR, pages 279-280, 1999.

10. N. Stokes, Y. Cavedon, and J. Zobel. Exploring criteria for succesful query expansion in the genomic domain. Information retrieval, 12:17-50, 2009.

11. J. Zhao, M. yen Kan, P. M. Procter, S. Zubaidah, W. K. Yip, and G. M. Li. Improving search for evidence-based practice using information extraction. $B M C$ Medical Informatics and Decision Making, 10(29), 2010. 